\title{
Cerebral Aspergillosis Presenting as a Mass Lesion
}

\author{
Negar Azarpira ${ }^{1}$, Majid Esfandiari ${ }^{1}$, Mohamad Hadi Bagheri ${ }^{2}$, Sed Rakei $^{3}$ and Soheila Salari ${ }^{4}$ \\ ${ }^{1}$ Transplant Research Center, Department of Pathology; ${ }^{2}$ Radiology Department; ${ }^{3}$ Neurosurgery Department, ${ }^{4}$ Internal Medicine Department; \\ Shiraz University of Medical Sciences; Shiraz, Iran
}

\begin{abstract}
Fungal infections of the central nervous system (CNS) are almost always a surprising finding. Their presentation is usually subtle, often without any diagnostic characteristics, and they are frequently mistaken for pyogenic abscesses, or brain tumors. Aspergillosis of the central nervous system is an uncommon infection, mainly occurring in immunocompromised patients. It may present in several forms, including meningitis, mycotic aneurysms, infarcts and a tumoral form. We report an intracranial granuloma due to Aspergillus fumigatus involving the anterior cranial fossa and the frontal lobe. The clinical symptoms began one year before admission. Final diagnosis was made after craniotomy. The patient was treated with an extensive excision of the cerebral mass and medical antifungal therapy (intravenous amphotericin B), but she failed to respond to these treatments and died.
\end{abstract}

Key-Words: Granulomatous inflammation, Aspergillus fumigatus, fungal infections.

Aspergillosis is a fungal infection caused by fungi of the genus Aspergillus. Aspergillus fumigatus is the most common human pathogen in the Aspergillus genus, but A. flavus and A. niger are also frequently found [1]. Aspergillus fungal spores are commensal in the respiratory tract and external auditory canal. The primary route of entry for aspergillosis pathogens is the respiratory tract. In the nervous system, this type of infection can be found in the cerebral parenchyma, the meninges or the vascular system [2,3]. Major risk factors for this disease include neutropenia and corticosteroid use. The most common presenting symptoms are nonspecific neurologic manifestations, including headache, cranial or somatic nerve weakness or paresthesia, altered mental status, and seizures. Aspergillosis as a cause of granulomatous inflammation of the brain is a rare condition [2,4]. Among its various presentations, a solitary intracranial mass is very uncommon [2,5]. Its preoperative diagnosis is very difficult. Because of its rarity in immunocompetent patients and the difficulty in preoperative diagnosis, we report a case found in Iran.

\section{Case Report}

A forty-nine-year-old diabetic woman was admitted in the Emergency Department of Chamran Hospital, affiliated with Shiraz University of Medical Sciences, Shiraz, in southern Iran. She had progressive headaches and anosmia during the previous four weeks. Her mental state was impaired, with no weakness or loss of vision. On neurological examination, the patient was confused and disoriented about time; but location and personal orientation remained intact. On fundoscopic examination, there was bilateral optic disc swelling (blurred disc margin in both eyes, more prominent in the left eye). On cranial nerve examination, there was anosmia of the left nostril. Other

Received on 3 April 2008; revised 21 July 2008.

Address for correspondence: Dr. Negar Azarpira, MD, Assistant Professor of Pathology, Transplant Research Center, Nemazee Hospital, Shiraz University of Medical Sciences, Shiraz, Iran. Phone/Fax: +98 7116276211. E-mail: negarazarpira@yahoo.com.

The Brazilian Journal of Infectious Diseases 2008;12(4):349-351. (C) 2008 by The Brazilian Journal of Infectious Diseases and Contexto Publishing. All rights reserved. aspects of neurological examination (muscle power, deep tendon reflexes, cerebellar tests, plantar and proprioceptive reflexes) were intact. A computed tomographic scan revealed a tumorlike lesion with high density and moderate mass in the frontal lobe, with edema in the surrounding area (Figure 1). It appeared to have moderate heterogeneous enhancement with contrast. The patient had a history of chronic sinusitis. Past medical history was negative for tuberculosis, bone marrow and solid organ transplantation and malignancy. There was no history of steroid or other immunosuppressive drug consumption. Immunological tests, including immunoglobulin levels (IgG IgM, IgA), as well as complete and differential blood counts, were within normal limits. Fasting blood sugar was $110 \mathrm{mg} / \mathrm{dL}$. A serological test for HIV was negative. The chest X-ray was normal. The patient underwent craniotomy. The lesion had a light yellowish color and a hard consistency. Pathologic study showed a granulomatous inflammation, with diffuse fibrosis that completely replaced normal brain tissue (Figure 2). Septate broad hyphae were easily demonstrated with periodic-acidSchiff (PAS) (Figure 3) and methenamine silver staining. Aspergillus fumigatus was isolated from a fungal culture of the brain tissue. During the postoperative period, the patient was treated with intravenous amphotericin $B$, but unfortunately the condition deteriorated and she died.

\section{Discussion}

Fungal infections have increased in frequency in the last decade because of the growing number of immunocompromised patients who survive longer periods than in the past, widespread use of immunosuppressive drugs, a large aging population with increased numbers of malignancies, and the spread of AIDS [6,7]. Mycotic diseases in the brain are usually secondary to infections elsewhere in the body, usually the lungs, less often from other extracranial sites, and in the vast majority of the cases are spread via blood circulation. Only occasionally, they result from direct extensions of infections of the sinuses or bones $[2,7,8]$. Most fungi cause basal meningitis or intraparenchymal abscesses. Direct extension from the cribriform plate can cause necrohemorrhagic lesions in the base of the frontal lobe. Although 
Figure 1. Mass-occupying lesion in frontal lobe (Computed tomographic scan).

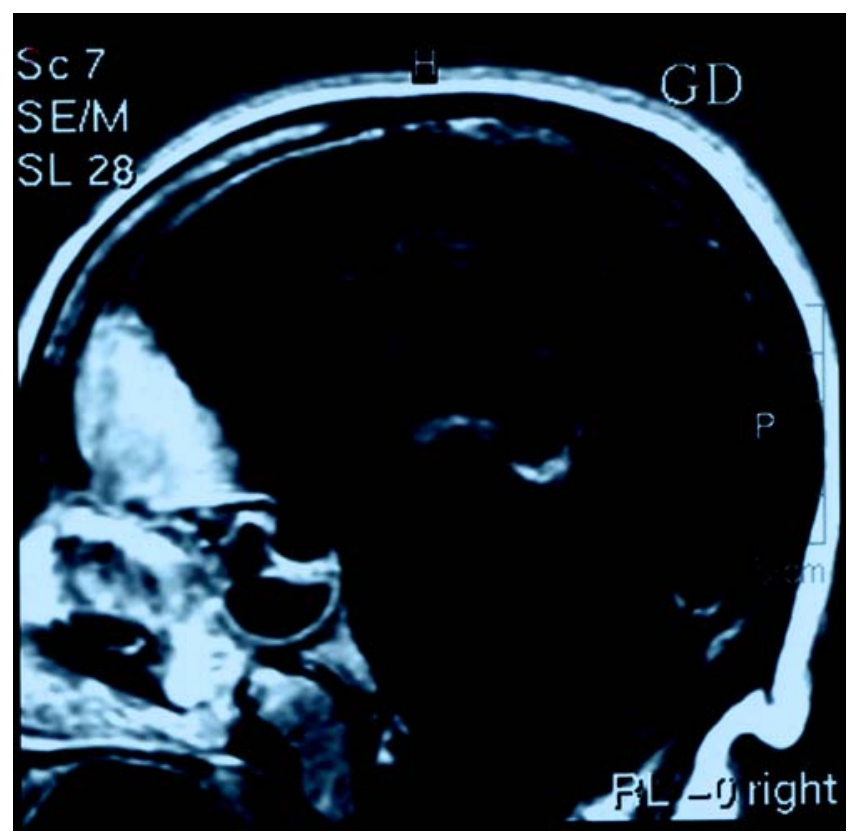

Figure 3. Septated hyphae (Periodic-Acid Schiff stain x 400).

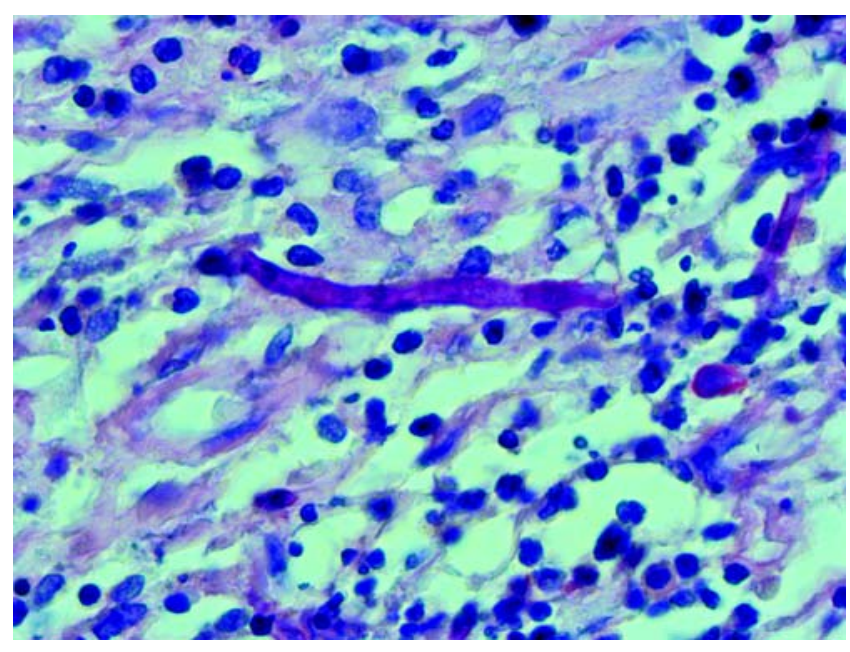

fungi are common in the environment, few are pathogenic. Candida, Cryptococus, Histoplasma, Aspergillus, Fusarium, Mucor and Rhizopus spp. can involve the central nervous system in special situations [1,8]. Aspergillosis should be considered in cases manifesting with acute onset of focal neurologic deficits resulting from a suspected vascular or spaceoccupying lesion $[1,2,6]$. Single or multiple abscess formation with blood vessel invasion and aneurysm formation are characteristic features of aspergillosis in neuropathologic examinations, especially in immunocompromised hosts. Aspergillus hyphae can invade directly into the vessel wall, resulting in mycotic aneurysm formation [6,7]. CNS aspergillosis
Figure 2. Non-caseating granulomatous inflammation replaces normal brain tissue. (H \& E x 400).

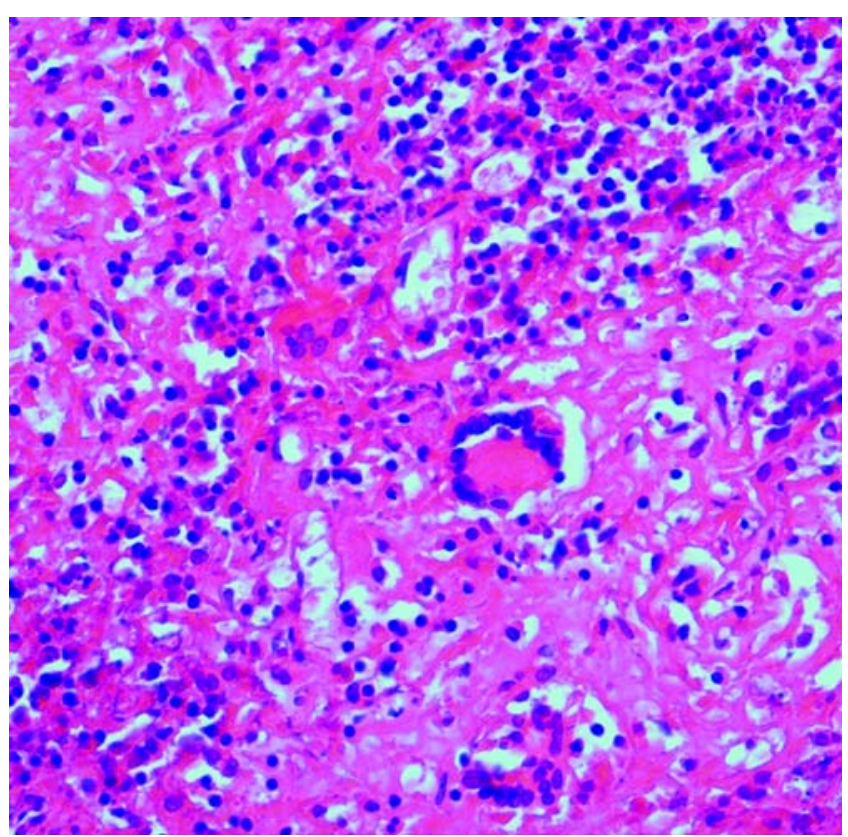

is also seen associated with cardiac, renal and other organ transplants [7]. Craniocerebral aspergillosis of sinonasal origin is also seen in immunocompetent patients [2]. The mechanism causing invasiveness of aspergillosis in immunologicallycompetent hosts remains unclear. It is possibly caused by qualitative cellular or subcellular immunodeficiency that is either unrecognized or poorly characterized [2]. Diagnosis of an intracranial mass lesion is best confirmed with computed tomography or magnetic resonance imaging of the head, with or without intravenous contrast. The differential diagnosis of a mass-occupying lesion with enhancement after contrast administration is brain tumors or abscesses.

Craniocerebral aspergillosis of sinonasal origin has typical MR imaging features. These features include a mass lesion producing hypo-to-iso-intense signals on T1-weighted, extremely low signals (hypo-intense) in T2-weighted images, with bright homogenous enhancement in post-gadolinium T1weighted imaging and disease processes in the nose and/or paranasal sinuses [11].

Positron emission tomography (PET) with ${ }^{18} \mathrm{~F}$ fluorodeoxyglucose (FDG) has also been widely used for differentiating between benign and malignant lesions, in surveys of recurrence and for evaluating therapeutic effects. PET/CT usually shows an increased FDG uptake in inflammatory lesions. If the necrotic nature of the disease is dominant, it might also contribute to the absence of hot uptake in PET/CT [12].

Our patient was operated based on the impression of a brain tumor. She was immunologically competent based on clinical evidence, as there was granuloma formation, which indicates a reaction of the immune system [2]. Nevertheless, 
diabetes mellitus and hyperglycemia affect the immune system. Diagnosis of aspergillosis is based on direct examinations and culture [8]. Aspergillus cultured optimally on Sabourad's agar produces characteristic conidiophores. In direct exam or pathology, Aspergillus is seen as septate branching hyphae, 4-12 $\mu$ m wide [1,6]. Surgical removal of Aspergillus abscesses and granulomas, as well as correction of underlying risk factors and antifungal treatment, are mandatory $[1,6]$.

The gold standard of systemic antifungal treatment is voriconazole, which has proven significantly superior to conventional amphotericin B and has led to a profound improvement of survival rates in patients with cerebral aspergillosis. Liposomal amphotericin B at standard dosages appears to be a suitable alternative for primary treatment, while caspofungin, amphotericin B lipid complex and posaconazole have given partial or complete cure in patients who are refractory to or intolerant of primary antifungal therapy. Combination therapies with two antifungal compounds could be a promising future strategy for firstline treatment [12]. According to Rogers (2008), studies of salvage therapy of zygomycosis with posaconazole have yielded promising results, and there are additional case reports of successful outcomes using these and other antifungal drugs as a combination therapy. Adjunctive approaches that are showing promise, but with limited clinical testing, include iron chelation and immunotherapy [13]. Only a high degree of suspicion, an aggressive approach to diagnosis and rapid vigorous therapy will alter the clinical course in this group of patients. The overall prognosis of craniocerebral aspergillosis is poor [2]. Mortality is reported to reach $100 \%$ in immunocompromised and $67 \%$ in immunocompetent hosts $[2,9,10]$.

Our case is an example of the rare presentation of aspergillosis; we emphasize the importance of suspecting this disease so that early pharmacotherapeutic intervention in addition to surgery can improve the prognosis of these patients.

\section{References}

1. Ruhnke M., Kofla G., Otto K., Schwartz S. CNS aspergillosis: Recognition, diagnosis and management. CNS Drugs 2007;21(8):659-76.

2. Siddiqui AA, Shah AA, Bashir SH. Craniocerebral aspergillosis of sinonasal origin in immunocompetent patients: Clinical spectrum and outcome in 25 cases. Neurosurgery 2004;55(3):602-11.

3. Tempkin A.D., Sobonya R.E., Seeger J.F., Oh E.S. Cerebral aspergillosis: radiologic and pathologic findings. Radiographics 2006;26(4):1239-42.

4. Haran R.P., Chandy M.J. Intracranial aspergillus granuloma. $\mathrm{Br} \mathrm{J}$ Neurosurg 1993;7(4):383-8.

5. Figueiredo E.G., Fonoff E., Gomes M., et al. Tumoral form of aspergillosis in central nervous system (cerebral aspergilloma): case report. Sao Paulo Med J 2003;121(6):251-3.

6. Marinovic T., Skrlin J., Vilendecic M., et al. Multiple Aspergillus brain abscesses in immuno-competent patient with severe craniofacial trauma. Acta Neurochir (Wien) 2007;149(6):629-32.

7. Murthy J.M., Sundaram C., Prasad V.S., et al. Aspergillosis of central nervous system: a study of 21 patients seen in a university hospital in south India. J Assoc Physicians India 2000;48(7):677-81.

8. Cho Y.S., Lee D.K., Hong S.D., Oh W.S. Intracranial aspergillosis involving the internal auditory canal and inner ear in an immunocompetent patient. AJNR Am J Neuroradiol 2007;28(1):138-40.

9. Sharma R.R., Gurusinghe N.T., Lynch P.G. Cerebral infarction due to Aspergillus arteritis following glioma surgery. Br J Neurosurg 1992;6:485-90.

10. Chernik N.L., Armstrong D., Posner J.B. Central nervous system infections in patients with cancer. Medicine 1973;52:563-81.

11. Siddiqui A.A., Bashir S.H., Ali Shah A., et al. Diagnostic MR imaging features of craniocerebral Aspergillosis of sino-nasal origin in immunocompetent patients. Acta Neurochir (Wien) 2006;148(2):155-66.

12. Maschmeyer G., Haas A., Cornely O.A. Invasive aspergillosis: epidemiology, diagnosis and management in immunocompromised patients. Drugs 2007;67(11):1567-601.

13. Rogers T.R. Treatment of zygomycosis: current and new options. J Antimicrob Chemother 2008;61:35-40. 